\title{
Noise Gated by Dendrosomatic Interactions Increases Information Transmission
}

\author{
Richard Naud, Alexandre Payeur, and André Longtin \\ Department of Physics, Center for Neural Dynamics, University of Ottawa, \\ 150 Louis Pasteur, Ottawa, Ontario, Canada, K1N 6N5 \\ and Department of Cellular and Molecular Medicine, University of Ottawa Brain and Mind Research \\ Institute, University of Ottawa, 451 Smyth Road, Ottawa, Ontario, Canada, K1H 8M5 \\ (Received 30 March 2017; revised manuscript received 13 July 2017; published 13 September 2017)
}

\begin{abstract}
We study how noise in active dendrites affects information transmission. A mismatch of both noise and refractoriness between a dendritic compartment and a somatic compartment is shown to lead to an inputdependent exchange of leadership, where the dendrite entrains the soma for weak stimuli and the soma entrains the dendrite for strong stimuli. Using this simple mechanism, the noise in the dendritic compartment can boost weak signals without affecting the output of the neuron for strong stimuli. We show that these mechanisms give rise to a noise-induced increase of information transmission by neural populations.

DOI: 10.1103/PhysRevX.7.031045

Subject Areas: Biological Physics, Complex Systems, Statistical Physics
\end{abstract}

\section{INTRODUCTION}

Biological systems process information with high efficiency despite a machinery characterized by high intrinsic variability. This incongruity may be resolved by considering the beneficial effects of noise on information transmission. At multiple time scales, noise can enhance information transmission for either subthreshold [1-3] or suprathreshold signals [4], a phenomenon known as aperiodic stochastic resonance. Yet, it is unclear how and to what extent neuronal populations exploit the noise inherent to the biophysics of membranes.

Intrinsic noise is known to contribute to the activity of single neurons [5-8]. It is thought to arise from stochastic changes in ion channel conformations regulating vesicle release and action potential generation. If such intrinsic noise were to play a constructive role in information transmission by either boosting subthreshold signals or decorrelating individual elements [1,4,9], its intensity should be tuned to the particular input $[10,11]$ : too little noise does not significantly enhance information, too much degrades it. It is unclear if single neurons can tune the intensity of intrinsic noise, and if so with what precision. Preferably, neurons would have a mechanism to gate noise selectively according to the strength of the input. Here, we study the extent with which noise in active dendrites affects the information transmitted by the cell body.

Dendrites are characterized by small compartment sizes [12-14], large intrinsic noise [15-17], and refractoriness

\footnotetext{
*rnaud@uottawa.ca
}

Published by the American Physical Society under the terms of the Creative Commons Attribution 4.0 International license. Further distribution of this work must maintain attribution to the author(s) and the published article's title, journal citation, and DOI.
$[18,19]$, which limits their maximal firing frequency. Conversely, the cell bodies of neurons are characterized by large compartment sizes, weak intrinsic noise, and can sustain high firing frequencies. These two types of neural subunits are active since they may generate spikes locally $[13,14,18,20]$. They are also coupled: a dendritic action potential can force a spike in the cell body and the backpropagating action potential couples the compartments in the reverse direction [12-14,18]. In this article, we show how these features can perform noise gating, and how this leads to an enhancement of time-dependent information transmission.

\section{DENDRITE-SOMA SYSTEM}

\section{A. Simplified biophysical description}

The role of dendrites can be addressed by a simplified biophysical model with a single dendritic compartment connected to the cell body. This dendrite-soma system is typically modeled with resistive coupling between the dendrite and the soma and a reduced set of ion channels on both compartments [21-23]. In an instantiation of such a system (see the Appendix), we simulate the response to a constant input delivered with equal strength to both compartments. In addition to this constant component, and to take into account noisier dendritic dynamics, the compartments are stimulated with independent noise scaled to have a 30-fold higher amplitude in the dendrite (see the Appendix). Figure 1 shows that the response consists of short and stereotypical action potentials in the soma, which are often associated with a broader action potential in the dendrite, consistent with experimental observations $[13,18,20]$ and detailed compartment modeling [20,24,25]. When the depolarizing input is weak, we find that a dendritic spike would consistently precede the somatic spike [Figs. 1(a) and $1(\mathrm{c})]$. In contrast, when the depolarizing input is strong, 
(a) Weak input

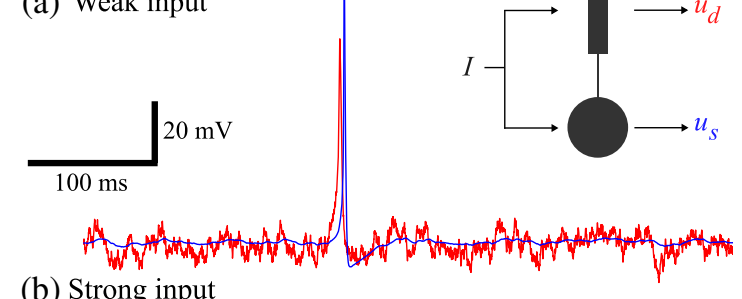

(b) Strong input
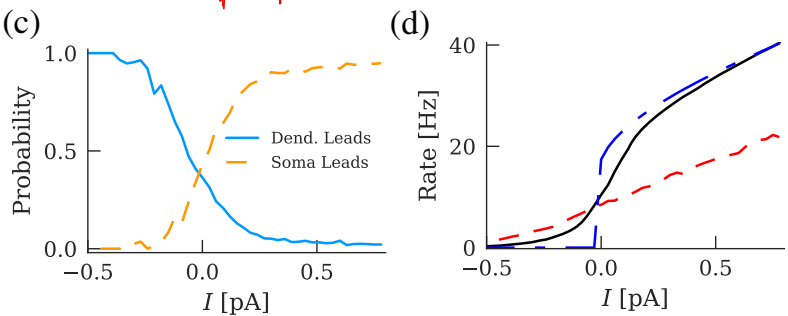

FIG. 1. Exchange of leadership in a simplified biophysical model of the dendrite-soma system. (a) The dendrite-soma system (see the Appendix) receiving a weak input in both the dendrite and the soma. The membrane potential of the soma (blue trace) and the dendrite (red trace) shows action potentials initiated first in the dendritic compartment. (b) For a strong input, the somatic compartment fires regularly and generally leads the dendritic action potentials. (c) The fraction of somatic spikes that are preceded by a dendritic spike within $8 \mathrm{~ms}$ (blue full line) and the fraction of dendritic spikes that are preceded by a somatic spike by at least $8 \mathrm{~ms}$ (orange dashed line) are shown. Spike timing is taken to be the time of crossing $-30 \mathrm{mV}$ from below. (d) The firing rate of the coupled system is shown as a function of the input strength (black full line). Isolating the compartments by fixing the coupling conductance to zero shows that the dendritesoma system interpolates between the isolated dendritic compartment (red dashed line) and the isolated somatic compartment (blue dash-dotted line).

the dendritic spike will generally follow the somatic spike [Figs. 1(b) and 1(c)]. In addition, we observe that the firing rate of the two-compartment system follows more closely the firing rate of an isolated dendritic compartment when the input is weak, and more closely the firing rate of an isolated somatic compartment when the input is strong [Fig. 1(d)].

\section{B. Integrate-and-fire description}

In order to identify central mechanisms from biophysical models, we use a simple yet accurate abstraction. We consider that the tip of dendrites can emit stereotypical spikes associated with a relative refractory period longer than that at the soma. We therefore model a dendrite-soma system as two interconnected integrate-and-fire units, a system studied in the context of connected pairs of neurons [26,27]. To model dendrite-soma systems, we consider that each compartment has independent intrinsic noise, a distinct refractory period, and common stimulation of intensity $s$. These effects are distinct from dendritic N-methyl D-Aspartate (NMDA) spikes [25,28-32], calcium spikes [21,22,33], or other simplified models of dendritic activity lacking either a back-propagating action potential or a clear refractory period $[34,35]$.

The dendritic (somatic) potential $u_{X}\left(u_{Y}\right)$ evolves according to the Langevin equations

$$
\begin{aligned}
& d u_{X}=\left(-u_{X}+s\right) d t / \tau+\sqrt{D_{X}^{2} / \tau} d W_{X}, \\
& d u_{Y}=\left(-u_{Y}+s\right) d t / \tau+\sqrt{D_{Y}^{2} / \tau} d W_{Y},
\end{aligned}
$$

with membrane time constant $\tau$ [36]. Upon reaching a threshold at $u_{X}\left(u_{Y}\right)>1$, a unit is said to fire. When a unit fires, it causes a strong potential jump $b$ in the other unit, consistent with strong active coupling between soma and dendrites [12-14,20]. This jump is implemented numerically in the time step after the firing time. After spiking, a unit remains clamped to the reset potential $V_{X}\left(V_{Y}\right)$ for unit $X(Y)$ during an absolute refractory period $T_{R}$, after which the membrane potential follows Eqs. (1) and (2). We model a different relative refractory period with different reset potentials since it takes a longer time to relax from a lower reset. We choose $V_{X}<V_{Y}$ to model a longer relative refractory period for the dendrite. Lastly, each unit is subjected to an intrinsic noise denoted by the independent Wiener increments $d W_{X}$ and $d W_{Y}$ in Eqs. (1) and (2) with intensity scaled by $D_{X}\left(D_{Y}\right)$. To comply with the intensity of intrinsic noise expected in neocortical dendrites [15-17], we consider that unit $X$ is noisier than unit $Y\left(D_{X}>D_{Y}\right)$ and study the dynamics of the system when $D_{X}$ is varied within a realistic range. Our analysis does not include an explicit subthreshold coupling between the compartments reflecting weak electrotonic coupling in the presence of active spike propagation in cortical dendrites [12-14]. With these parameter restrictions, the noisier and more refractory unit $(X)$ models an active dendrite while unit $Y$ corresponds to the soma, and so it is the output of the system.

\section{EXCHANGE OF LEADERSHIP AS NOISE GATING}

\section{A. Exchange of leadership}

The coupling between the units implies that whenever one of the units fires, the other has a high probability to discharge immediately afterwards. The relative refractory period prevents another firing event to directly follow this dual firing. Between these dual spiking events the units are effectively independent. Then, the first unit reaching threshold dictates the firing dynamics of the coupled 

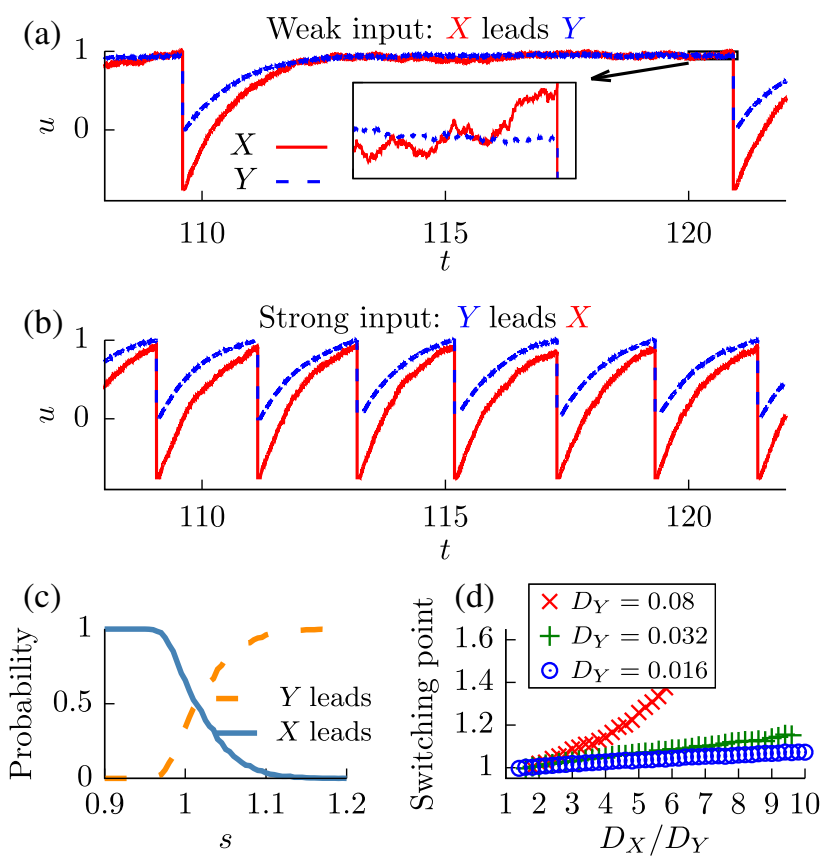

FIG. 2. Noise gating in coupled integrate-and-fire units. (a) Membrane potential response of $Y$ (somatic, blue dashed line) and $X$ (dendritic, red full line) units for $s=0.95$ and $D_{X}=3 D_{Y}$. Inset: Expanded view near threshold, where the $X$ unit crosses first (arrow). (b) Same as (a) but for stronger input $s=1.15$. (c) Entrainment probability for a range of input strengths, as described in the caption of Fig. 1 The time window used to establish leadership in the integrate-and-fire model is of the same order as the integration time step. The input intensity where entrainment probabilities cross is the switching point. (d) Dependence of the switching point on $D_{X} / D_{Y}$ for different values of $D_{Y}$. Parameters are described in [36].

system. In the subthreshold regime, i.e., for $s<1$, the potential of each unit cannot cross threshold without noise, and both $u_{X}$ and $u_{Y}$ would saturate to $s$ in this case. At this potential, the noisier unit has a greater probability to fire since $D_{X}>D_{Y}$. The noisier unit $X$ will fire more often and therefore be the leader, entraining the more deterministic unit $Y$ as illustrated in Fig. 2(a). On the other hand, in the suprathreshold regime $(s>1)$, both units fire without noise. Hence, following a spike, unit $Y$ can take advantage of its higher reset value and cross the threshold before $X$ [Fig. 2(b)].

An exchange of leadership, or switching, occurs as $s$ is varied from subthreshold to suprathreshold values. We compute the probability that $X$ entrains $Y, P_{X \rightarrow Y}$, by counting the fraction of $X$ spikes that are immediately followed by a $Y$ spike. Figure 2(c) shows $P_{X \rightarrow Y}$ and its complement $P_{Y \rightarrow X}$ calculated by numerical simulation of Eqs. (1) and (2) in a typical example of switching. The dendritic leadership is complete and sustained at subthreshold input strengths. As $s$ is increased, dendritic leadership is diminished and $P_{X \rightarrow Y}$ decreases. Simultaneously, somatic leadership is augmented and $P_{Y \rightarrow X}$ increases. In the strong input regime the exchange of leadership is complete such that $P_{X \rightarrow Y}$ reaches zero and $P_{Y \rightarrow X}$ one.

We observe complete switching provided that the relative dendritic noise $D_{X}$ is sufficiently greater than $D_{Y}$ and sufficiently small compared to the coupling amplitude $b$ to ensure strong effective coupling. Within this range, the switching point defined by $P_{X \rightarrow Y}=P_{Y \rightarrow X}=0.5$ is not constant but increases with $D_{X}$ [Fig. 2(d)]. A mismatch of the level of noise across compartments combined with a mismatch of refractoriness can therefore mediate the inputdependent exchange of leadership.

\section{B. Noise gating}

Since the switching seen at strong inputs implies that the influence of high intrinsic noise in unit $X$ is removed from the output, we remark that the system effectively reduces, or gates, noise as a function of input intensity. Furthermore, gating emerges close to the deterministic threshold, precisely at the point where the role of noise switches from beneficial to detrimental in single-unit encoding. It suggests a particular role for noise gating: a more deterministic encoding of suprathreshold inputs and a noise-assisted encoding to resolve subthreshold inputs within the same encoding device and without the use of feedback.

\section{EFFECTS ON ENCODING PRECISION}

\section{A. Stationary inputs}

To show the role of a dendrosomatic mismatch of noise on encoding quality, we investigate the consequences of noise gating on stationary firing statistics (Fig. 3). For various input strengths $s$, we compute the mean firing rate and the variance of the interspike intervals $\left(\sigma_{\text {ISI }}^{2}\right)$. At subthreshold input strengths, the firing rate of the coupled system is identical to the firing rate of an uncoupled $X$ unit. In this regime, the dendritic compartment controls the timing of the somatic compartment, consistent with in vitro recordings [37] and our biophysical model [Fig. 1(a)]. As the input strength is increased, the firing rate of the coupled system starts to deviate from that of an uncoupled $X$ unit, reaching the firing rate predicted for an isolated $Y$ unit when switching is complete [Fig. 3(a)]. Interspike interval variance similarly switches from a variability predicted by the dynamics of an $X$-unit subthreshold to a variability predicted by the dynamics of a $Y$-unit suprathreshold [Fig. 3(b)], as is to be expected from the gating of $X$-unit noise. Notably, the coupled system follows the strongest firing rate and the smallest variability, concurrently.

To determine if switching affects signal encoding, we calculate the Fisher information that interspike intervals $T$ carry about a constant input. Fisher information measures how sensitive an observable such as the interspike interval $T$ is to changes in an input parameter $s$. In practice, we use an approximation to the Fisher information rate, 

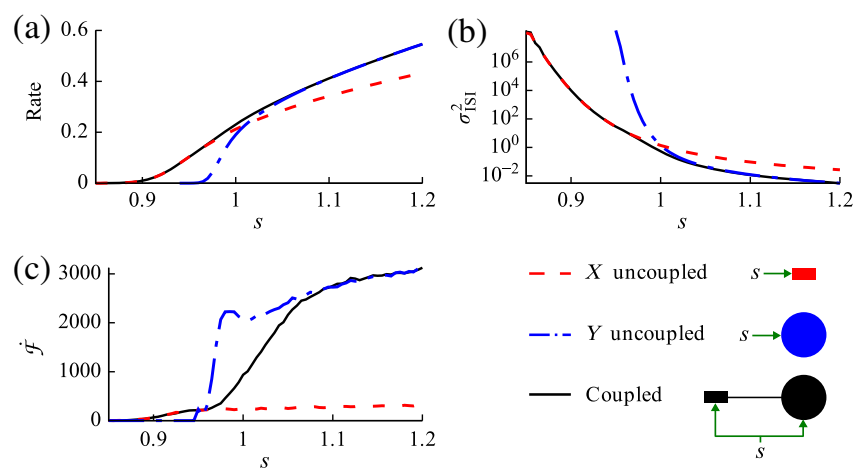

FIG. 3. The firing statistics of isolated units constitute asymptotic curves for the dendrite-soma system. (a) Firing rate of coupled system (black line), $X$ unit alone (i.e., dendrite alone, red line), and $Y$ unit alone (i.e., soma alone, blue line) for different input strengths. (b) The interspike interval variance of the coupled system follows the minimum between isolated $X$ and $Y$ units. (c) Fisher information about the input strength. Parameters are described in [36].

$$
\dot{\mathcal{F}}(s)=\frac{\mu_{T}^{\prime}(s)^{2}}{\mu_{T}(s) \sigma_{T}^{2}(s)},
$$

where $\mu_{T}^{\prime}$ is the derivative of the mean interspike interval with respect to the mean input $s$. The quantity described by Eq. (3) is widely used in studies of neural coding since it is a lower bound on the Fisher information of a population of spiking neurons with independent noise [38,39].

Consistent with the firing rate and interval variability we describe above, $\dot{\mathcal{F}}$ of the coupled system is predicted by $X$-unit properties subthreshold and by $Y$-unit properties after complete switching. In the limit of both small and large input strengths, the coupled system shows $\dot{\mathcal{F}}$ as high as the maximum between isolated $X$ or $Y$ units [Fig. 3(c)]. Since for $s<1 \dot{\mathcal{F}}$ is enhanced by noise, we observe that the stochastic enhancement due to $X$-unit noise is preserved in the dendrite-soma system. Near threshold, however, the coupled system shows lower $\dot{\mathcal{F}}$ than the isolated $Y$ unit. To summarize, Fig. 3 suggests that noise gating is beneficial for encoding either weak or strong signals, but not input strengths that lie predominantly close to the deterministic threshold. For these perithreshold inputs, the isolated $Y$ unit translates small input increments into consistently strong firing rate changes while the coupled system randomly switches between $X$ - and $Y$-driven firing with similar rates.

\section{B. Time-dependent inputs}

These observations suggest that if a time-dependent input is less often around threshold values, while sampling more consistently both subthreshold and suprathreshold input intensities, then noise gating could enhance the encoding of time-dependent inputs. To achieve this, the coupled system would rely on noisier unit $X$ when the input is below the deterministic threshold at $s=1$. When the input is above the deterministic threshold, the system would switch to a more deterministic encoding by relying on $Y$ units. Therefore, we hypothesize that noise gating can enhance encoding of a time-dependent input, even for inputs distributed predominantly above threshold. Additionally, the enhancement should be robust for large dendritic noise since strong noise does not degrade the suprathreshold part of the signal due to noise gating.

To test this hypothesis, we simulate 8000 dendrite-soma systems receiving the same time-dependent input $s(t)$ with mean $\bar{s}>1$, in the regime of suprathreshold stochastic resonance [4]. An estimate of the population activity is constructed by summing the 8000 spike trains from all $Y$ units. Encoding quality is quantified by Shannon's information for the classic channel with additive Gaussian noise [40-42],

$$
\mathcal{M}=-\int_{0}^{\infty} \log _{2}[1-C(f)] d f
$$

where $C(f)$ is the coherence between the population activity and the input $s(t)$ for each frequency $f$ [43]. $\mathcal{M}$ encapsulates the frequency-resolved measure of correlation between input and output fluctuations $C(f)$ into a single quantity. This quantity is distinct from the average firing rate, and it is used as a lower bound on the mutual information between the time-dependent input and the set of spike trains.

Figure 4 shows $\mathcal{M}$ for increasing intrinsic dendritic noise and two types of input currents $s(t)$. For the first type, we consider a jump-diffusion process (JDP) producing random fluctuations around two states with random switching times between the high and the low states. The JDP parameters [44] are chosen to produce a bimodal distribution centered slightly above threshold such that the switching point [Fig. 2(d)] may cross the center of the input distribution as $D_{X}$ is varied [Fig. 4(a)]. Although the exact mutual information has not yet been derived for JDPs, this process is chosen to ensure a bimodal distribution of inputs without imposing a periodic structure. It is a physiologically realistic input distribution since sinusoidal sensory inputs and up and down states are frequently treated in the context of neuroscience [45-47]. We compare JDP encoding with encoding of a Gaussian process (GP) simulated with the Euler-Maruyama method with matched mean and variance [Fig. 4(b)], focusing, as a first step, on narrow input distributions.

For narrow input distributions, a resonance as a function of $D_{X} / D_{Y}$ is seen for the JDP but not the GP [Figs. 4(d) and 4(e)]. Consistent with a more frequent sampling of elevated $\dot{\mathcal{F}}$ perithreshold [48], $\mathcal{M}$ is generally higher for the unimodal input than for the bimodal input. At the maximum, or resonance, and only for the bimodal input, the coupled system surpasses in encoding quality a more deterministic population made of isolated $Y$ units 
(a)

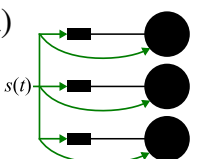

(b)

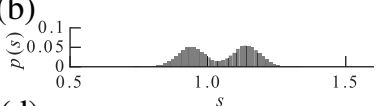

(d)

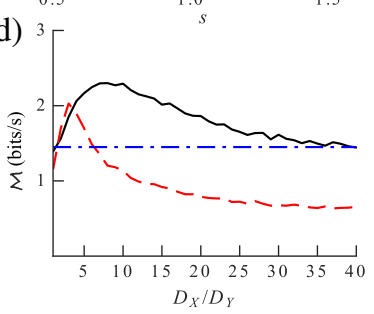

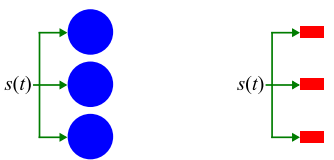

(c)

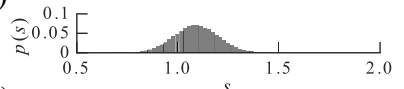

(e)

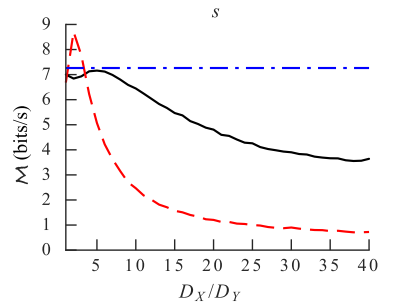

FIG. 4. Stochastic resonance for bimodal input distributions. (a) Schema illustrating a neural ensemble made of dendrite-soma systems (black), somatic units alone (blue), or dendritic unit alone (red). Input distribution for (b) the JDP and (c) a GP with mean matched to that of the JDP. (d) For the JDP, $\mathcal{M}$ calculated from the summed activity of 8000 coupled units (black line) surpasses that of isolated $Y$-unit compartments (blue dot-dashed line) and of isolated dendritic compartments (red dashed line) for a broad range of dendritic noise. (e) The resonance for a GP is reduced with respect to the resonance of obtained using the JDP (d). We use $\tau=10 \mathrm{~ms}$ to represent $\mathcal{M}$ in bits per second; see [36] for all other parameters.

[dash-dotted line in Figs. 4(d) and 4(e)]. This stochastic enhancement is seen for a large range of intrinsic noise. The coupled system also surpasses a population of isolated $X$ units with the corresponding intrinsic noise intensity, again only for bimodal inputs. The resonance in Fig. 4(d) can be understood by recalling that two competing processes result from an increase in $D_{X}$. On the one hand, weaker signals can trigger spikes, which increases the coding range and therefore $\mathcal{M}$. On the other hand, the switching point increases, decreasing the range of inputs encoded by the $Y$ unit [Fig. 2(d)], which decreases reliability and therefore $\mathcal{M}$. The output unit (unit $Y$ ) representation of bimodal inputs can therefore be enhanced by noise in an auxiliary unit (unit $X$ ). The optimal noise results from a trade-off between increasing the coding range and decreasing the coding reliability.

The particular scenario illustrated in Fig. 4(c) corresponds to a coding enhancement with respect to the isolated $Y$ units of up to $61 \%$ in $\mathcal{M}$ [Fig. 4(c)]. Finite-size effects deteriorate this enhancement, which can be verified by halving the population size. This results in a reduction of the enhancement to $42 \%$ in $\mathcal{M}$. We find that the enhancement in $\mathcal{M}$ is not detectable for a population of 250 coupled systems, but is present for a population of 500 coupled systems. The enhancement holds for sinusoidal inputs, which can be verified by using a sinusoidal input with matched bimodal peaks. Furthermore, the enhancement necessitates a paucity of perithreshold input strengths, which can be verified by simulating a JDP with a mean

(a)

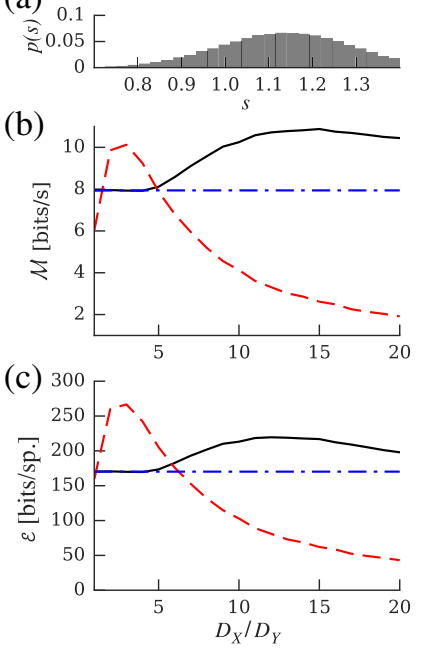

(d)

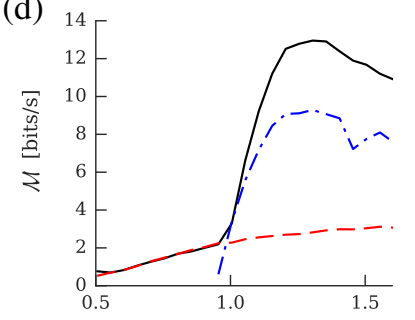

(e) 200

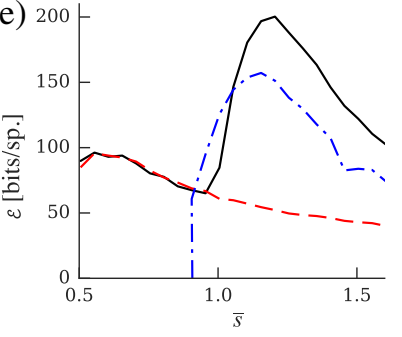

FIG. 5. Stochastic resonance for wide input distributions. (a) Input distribution of the GP chosen to cover a large range around a high mean $\bar{s}$. Using the same color code as in Fig. 3, (b) shows $\mathcal{M}$ and (c) shows $\mathcal{E}$ (in bits per spikes) for different $D_{X}$ calculated with input distribution in (a). We restrict the range for intrinsic noise such that the standard deviation of $u_{X}$ remains smaller than 0.5 , which would correspond to substantial membrane potential fluctuations in physical units. (d) $\mathcal{M}$ and (e) $\mathcal{E}$ for different values of the mean input and for a fixed level of intrinsic noise $D_{X}=15 D_{Y}$. Parameters are described in [36].

increased from 1.04 to 1.14 , such that the leftmost peak is close to the deterministic threshold. This manipulation removes the stochastic enhancement. We conclude that intrinsic dendritic noise, when gated by input intensity, may improve $\mathcal{M}$ for inputs rarely lying close to threshold. Also, this enhancement is more pronounced in range and amplitude than with classical mechanisms for stochastic resonance [1-4] [see red curve in Fig. 4(d)].

In the context of neuroscience, the inputs are likely to be broadly distributed. We thus consider a broad GP input distribution with twice the standard deviation of the distribution shown in Fig. 4. We find that even when the average input is above threshold [Fig. 5(a)], the coupled system shows stochastic enhancement over a large range of intrinsic noise levels [Fig. 5(b)]. Since $\mathcal{M}$ remains high even at the largest levels of noise we consider, the dendritesoma system can tolerate noise coming in addition to noise expected from intrinsic sources [15-17]. Background synaptic noise and finite-size noise are examples of extrinsic noise sources that could contribute to this enhancement.

To verify that the information enhancement is not simply due to a rate increase, we normalize $\mathcal{M}$ by the average firing rate $\nu$ and consider the quantity

$$
\mathcal{E}=\frac{\mathcal{M}}{r+\nu} .
$$

Since every spike comes with a metabolic energy cost, $\mathcal{E}$ is interpreted as a measure of energy efficiency of information 
transfer $[49,50]$. We report here a quantity in bits per spike, but this can be converted into bits per adenosine triphosphate (ATP) molecule using numerical estimates of the number of ATP molecules used for the generation of an action potential [49]. The parameter $r$ is interpreted as the firing rate at which the energy expenditure of action potential generation equals the energy expenditure of maintaining a depolarized membrane potential. This parameter is thought to vary substantially across neuron types [51], but to be relatively low (we fix $r=5 \mathrm{~Hz}$ ). Figure 5(c) shows an enhanced efficiency for large dendritic noise in the coupled system. In addition, the efficiency of the coupled system surpasses the efficiency of either a population of somatic compartments or a population of dendritic compartments with matched level of intrinsic noise.

We now ask how enhancement depends on the mean input. The $\mathcal{M}$ of the coupled system matches that of isolated $X$ units when the mean is subthreshold [Fig. 5(d)], but surpasses those of isolated $X$ and $Y$ units when the mean is suprathreshold. Therefore, for broadly distributed inputs, noise gating allows stochastic enhancement on a broad range of intrinsic noise levels, thus alleviating the need for precise noise tuning in neurons. The energy efficiency is similarly enhanced [Fig. 5(e)], but shows two peaks: a first peak subthreshold, matching the enhancement obtained by isolated dendritic units, and a second, much higher peak suprathreshold, exceeding the efficiency of isolated somatic units. These results are consistent with the enhanced $\mathcal{M}$ [Fig. 5(d)] and a firing rate that follows the largest of either unit [Fig. 1(d) and Fig. 3(a)]. Hence, noise gating in dendrite-soma systems allows an efficient encoding through a stochastic enhancement of subthreshold signals that preserves deterministic encoding suprathreshold.

\section{DISCUSSION}

Several other theoretical studies pointed to a functional relevance of spiking or nonlinear summation in dendrites. One view holds that dendrites function as sigmoidal units $[28,29]$. This simple description was shown to capture the time-average firing rate of biophysical neuron models with detailed morphology and active dendritic conductances supporting NMDA spikes [25,31,52]. Modeling studies have shown that this architecture provides multiple advantages, namely, specific sensory computations [53], enhanced memory capacity [30,35], enhanced dynamic range [54,55], and flexible gating of specific pathways [32]. These computational advantages are based on a phenomenological description of the time-averaged firing rate, which could remain consistent with the timingdependent mechanisms we describe here. Additionally, the timing-dependent network synchrony mechanism discussed in Ref. [34] is likely to hold in the presence of noise gating. Therefore, we add to the known computational advantages of nonlinear dendrites an improvement of encoding precision based on spike-timing interactions.

Experimental studies point to a surprising diversity of active dendrites. The mechanism we describe here is likely to remain relevant when a combination of long refractory period, small subthreshold coupling, and large noise is achieved. We argue that high intrinsic noise and small subthreshold coupling is expected in thin basal dendrites more than one electrotonic constant away from the cell body. First, from measurements of dendrite diameter at the tip of dendrites $(0.5-0.6 \mu \mathrm{m}[56,57])$ and experimental estimates of the effective length of a compartment ( $250 \mu \mathrm{m}$ [20]), the effective dendritic surface area is estimated to be approximately $400-500 \mu \mathrm{m}^{2}$. Second, theoretical studies [16] predict that a compartment with surface area of $500 \mu \mathrm{m}^{2}$ would exhibit noisy fluctuations with standard deviation of $1 \mathrm{mV}$ from the stochastic opening of ion channels, a tenfold increase from observed somatic noise [58] (consistent with our $D_{X} / D_{Y} \sim 10$ ). Such basal dendrites have accrued refractoriness [18,19], and longer action potentials [20,59]. Therefore, the known properties of thin basal or oblique dendrites are consistent with the mechanisms we describe here.

Other dendritic compartments may implement different functions. Notably, the apical tuft of cortical pyramidal cells is known to generate calcium regenerative events, which lead to a burst of action potentials $[60,61]$ and a dendritic control on somatic gain $[62,63]$. In addition, interactions between synaptic plasticity and dendritic dynamics have been the focus of much recent attention [32,64-66]. In summary, although these mechanisms are not immediately compatible with those we describe here, they can be combined in a juxtaposition of distinct, spatially segregated compartments within the same dendritic tree.

If noise gating is taking place in the nervous system according to the mechanism we describe in this article, we predict that spikes encoding a weak stimulus would have been initiated more frequently by the dendrites, whereas spikes encoding a strong stimulus would have been initiated more frequently at the soma. Given experimental evidence indicating a connection between the shape of the action potential and the site of initiation $[14,67]$, it appears possible to test this prediction experimentally. Furthermore, focal pharmacological manipulations can be used to determine the role of active dendritic conductances for different strengths of sensory stimulation $[68,69]$.

As a second prediction, we note that the dendritic refractory period should be substantially greater than in the soma for the switching point to be in the typical range of firing rate. Although there are clear indications that the dendrite is more refractory to spiking $[18,19]$, we are not aware of a direct measurement showing a longer relative or absolute refractory period in the dendrites. A potent discrepancy is likely, given the different composition of 
ion channels in dendrites [70]. Noting that lengthening of the relative refractory period may arise from either a spiketriggered hyperpolarization or a spike-triggered increase in the action potential threshold [71], an electrophysiology experiment $[13,20,37,60]$ can be designed to measure the difference between somatic and dendritic refractory processes.

\section{CONCLUSION}

The mechanism we outline in this article may provide a functional role of intrinsic noise in active dendrites, where the dendrite act as a noise-assisted encoder, acting only at small firing rates. Also, we describe how the effects of intrinsic noise in a subsystem can be gated by a mismatch of refractory periods between compartments. To conclude, we outline a novel mechanism for information enhancement by intrinsic noise. This coding strategy allows us to communicate substantially more information per action potential, an interesting approach given the metabolic costs of action potential transmission. Because the mechanism is simple and easy to implement, it can inspire novel engineering approaches to signal detection.

\section{ACKNOWLEDGMENTS}

We thank Tilo Schwalger, Jean-Claude Béique, and Len Maler for helpful discussions, as well as FRQNT postdoctoral scholarships (R.N.) and the NSERC Discovery Grants (A. L. and R. N.) for funding.

\section{APPENDIX: SIMPLIFIED BIOPHYSICAL MODEL OF THE DENDRITE-SOMA SYSTEM}

We model the soma-dendrite system as two connected electrical compartments with different densities of voltagegated ion channels. We note that this biophysical description is lacking multiple biophysical details, namely, multiple dendrites, impedance mismatch between soma and dendrite, multiple other types of ion channels, and active or quasiactive propagation. Yet, this two-compartment abstraction has been instrumental for understanding many features of dendritic computation $[21,22,62,72,73]$.

The somatic membrane potential $u_{s}$ and the dendritic membrane potential $u_{d}$ evolve according to Kirchoff's circuit law for the conservation of current,

$$
\begin{aligned}
& C \frac{d u_{s}}{d t}=-g_{L}\left(u_{s}-E_{L}\right)-\sum_{i} I_{i}^{(s)}-g_{c}\left(u_{s}-u_{d}\right)+I_{\mathrm{ext}}^{(s)}, \\
& C \frac{d u_{d}}{d t}=-g_{L}\left(u_{d}-E_{L}\right)-\sum_{i} I_{i}^{(d)}-g_{c}\left(u_{d}-u_{s}\right)+I_{\mathrm{ext}}^{(d)},
\end{aligned}
$$

where $C=0.75 \mathrm{pF}$ is the compartment capacitance, $g_{L}=$ $0.2 \mathrm{nS}$ is the leak conductance, and $E_{L}=-70 \mathrm{mV}$ is the leak reversal potential. The two compartments are coupled via the coupling conductance $g_{c}=0.02 \mathrm{nS}$.

Each compartment contains a different set of voltagegated ion channels. The soma is modeled with a combination of inactivating sodium conductance $I_{\mathrm{Na}}$ and fast rectifying potassium conductance $I_{\mathrm{K}}$ :

$$
\sum_{i} I_{i}^{(s)}=I_{\mathrm{Na}}^{(s)}+I_{\mathrm{K}}^{(s)}
$$

The sodium conductance follows the Hodgkin-Huxley kinetics with an activation gate $m$ and an inactivation gate $h, I_{\mathrm{Na}}=g_{\mathrm{Na}}^{(s)} m^{3} h\left(u_{s}-E_{\mathrm{Na}}\right)$, where $g_{\mathrm{Na}}^{(s)}=20 \mathrm{nS}$ is the maximal conductance and $E_{\mathrm{Na}}=60 \mathrm{mV}$ is the reversal potential for sodium. The potassium conductance is modeled with a single activation gate $I_{\mathrm{K}}=g_{\mathrm{K}}^{(s)} n\left(u_{s}-E_{\mathrm{K}}\right)$, where $g_{\mathrm{K}}^{(s)}=10 \mathrm{nS}$ is the maximal conductance and $E_{\mathrm{K}}=$ $-70 \mathrm{mV}$ is the reversal potential for potassium. The kinetics of the gating variables $x \in\{m, h, n\}$ follows $\tau_{x}\left(u_{s}\right) \dot{x}=x_{0}\left(u_{s}\right)-x$, with $\tau_{x}(u)$ and $x_{0}(u)$ described in Table I. The dendrite contains these two types of ion channels, but with lower densities:

$$
\sum_{i} I_{i}^{(d)}=I_{\mathrm{Na}}^{(d)}+I_{\mathrm{K}}^{(d)}
$$

In the dendritic compartment, the maximal conductance of sodium is $g_{\mathrm{Na}}^{(d)}=10 \mathrm{nS}$ and the that of potassium to $g_{\mathrm{K}}^{(d)}=8.0 \mathrm{nS}$. The kinetics of the gating variables $x \in$ $\{m, h, n\}$ follows $\tau_{x}\left(u_{d}\right) \dot{x}=x_{0}\left(u_{d}\right)-x$, with $\tau_{x}(u)$ and $x_{0}(u)$ described in Table I.

TABLE I. Hodgkin-Huxley kinetics for three types of voltage-gated ion channels. Parametrization follows experiments in neocortical neurons [74-76].

\begin{tabular}{lcc}
\hline \hline Symbol & $\tau_{x}(u)[\mathrm{ms}]$ & $x_{\infty}(u)$ \\
\hline$m$ & $\{1-\exp [-(u-35 \mathrm{mV}) / 9 \mathrm{mV}]\} /((u-35 \mathrm{mV})(0.182+0.124)$ & $0.182 /[0.182+0.124$ \\
& $\times\{1-\exp [-(u-35 \mathrm{mV}) / 9 \mathrm{mV}]\})$ & $\times\{1-\exp [-(u-35 \mathrm{mV}) / 9 \mathrm{mV}]\})$ \\
$h$ & $109 /(2.6(u+50 \mathrm{mV}) /\{1-\exp [-(u+50 \mathrm{mV}) / 5 \mathrm{mV}]\}$ & $1 /\{1+\exp [(u+65 \mathrm{mV}) / 6.2 \mathrm{mV}]\}$ \\
& $-(u+75 \mathrm{mV}) /\{1-\exp [(u+75 \mathrm{mV}) / 5 \mathrm{mV}]\})$ & $0.02 /(0.02+0.002$ \\
$n$ & $\{1-\exp [-(u-25 \mathrm{mV}) / 9 \mathrm{mV}]\} /((u-25 \mathrm{mV})(0.02+0.002$ & $\times\{1-\exp [-(u-25 \mathrm{mV}) / 9 \mathrm{mV}]\})$ \\
& $\times\{1-\exp [-(u-25 \mathrm{mV}) / 9 \mathrm{mV}]\}))$ & \\
\hline \hline
\end{tabular}


Each compartment receives an external input that is partitioned into three terms:

$$
\begin{aligned}
& I_{\mathrm{ext}}^{(s)}=I_{\mathrm{rh}}+I+\sigma_{s} \xi^{(s)}, \\
& I_{\mathrm{ext}}^{(d)}=I_{\mathrm{rh}}+I+\sigma_{d} \xi^{(d)} .
\end{aligned}
$$

The term $I_{\mathrm{rh}}=0.57 \mathrm{pA}$ is a constant current corresponding to the rheobase of the system. The additional current $I$ controls the current injected into the compartments with respect to this rheobase. We also include independent background noises $\xi^{(s)}$ and $\xi^{(d)}$ drawn from a normal distribution $[\sim \mathcal{N}(0,1)]$ independently at every time step of size $d t=0.1 \mathrm{~ms}$ and for each compartment. The noise amplitude is scaled by $\sigma_{s}=0.1 \mathrm{pA}$ in the soma and $\sigma_{d}=3 \mathrm{pA}$ in the dendrite.

[1] J. Collins, C. C. Chow, and T. T. Imhoff, Stochastic Resonance without Tuning, Nature (London) 376, 236 (1995).

[2] D. R. Chialvo, A. Longtin, and J. Müller-Gerking, Stochastic Resonance in Models of Neuronal Ensembles, Phys. Rev. E 55, 1798 (1997).

[3] P. C. Gailey, A. Neiman, J. J. Collins, and F. Moss, Stochastic Resonance in Ensembles of Nondynamical Elements: The Role of Internal Noise, Phys. Rev. Lett. 79, 4701 (1997).

[4] N. G. Stocks, Suprathreshold Stochastic Resonance in Multilevel Threshold Systems, Phys. Rev. Lett. 84, 2310 (2000).

[5] A. Longtin, A. Bulsara, and F. Moss, Time-Interval Sequences in Bistable Systems and the Noise-Induced Transmission of Information by Sensory Neurons, Phys. Rev. Lett. 67, 656 (1991).

[6] Z. F. Mainen and T. J. Sejnowski, Reliability of Spike Timing in Neocortical Neurons, Science 268, 1503 (1995).

[7] W. C. Stacey and D. M. Durand, Stochastic Resonance Improves Signal Detection in Hippocampal CA1 Neurons, J. Neurophysiol. 83, 1394 (2000).

[8] C. M. Marcoux, S. E. Clarke, W. H. Nesse, A. Longtin, and L. Maler, Balanced Ionotropic Receptor Dynamics Support Signal Estimation via Voltage-Dependent Membrane Noise, J. Neurophysiol. 115, 530 (2016).

[9] M. D. McDonnell and D. Abbott, What is Stochastic Resonance? Definitions, Misconceptions, Debates, and Its Relevance to Biology, PLoS Comput. Biol. 5, e1000348 (2009).

[10] S. Mitaim and B. Kosko, Adaptive Stochastic Resonance, Proc. IEEE 86, 2152 (1998).

[11] G. Wenning and K. Obermayer, Activity Driven Adaptive Stochastic Resonance, Phys. Rev. Lett. 90, 120602 (2003).

[12] G. J. Stuart and B. Sakmann, Active Propagation of Somatic Action Potentials into Neocortical Pyramidal Cell Dendrites, Nature (London) 367, 69 (1994).

[13] N. L. Golding and N. Spruston, Dendritic Sodium Spikes Are Variable Triggers of Axonal Action Potentials in
Hippocampal CA1 Pyramidal Neurons, Neuron 21, 1189 (1998).

[14] S. L. Smith, I. T. Smith, T. Branco, and M. Häusser, Dendritic Spikes Enhance Stimulus Selectivity in Cortical Neurons In Vivo, Nature (London) 503, 115 (2013).

[15] G. Schmid, I. Goychuk, and P. Hänggi, Stochastic Resonance as a Collective Property of Ion Channel Assemblies, Europhys. Lett. 56, 22 (2001).

[16] C. O'Donnell and M. C. van Rossum, Systematic Analysis of the Contributions of Stochastic Voltage Gated Channels to Neuronal Noise, Front. Comput. Neurosci. 8, 105 (2014).

[17] R. C. Cannon, C. O'Donnell, and M. F. Nolan, Stochastic Ion Channel Gating in Dendritic Neurons: Morphology Dependence and Probabilistic Synaptic Activation of Dendritic Spikes, PLoS Comput. Biol. 6, e1000886 (2010).

[18] C. M. Colbert, J. C. Magee, D. A. Hoffman, and D. Johnston, Slow Recovery from Inactivation of $\mathrm{Na}^{+}$Channels Underlies the Activity-Dependent Attenuation of Dendritic Action Potentials in Hippocampal CA1 Pyramidal Neurons, J. Neurosci. 17, 6512 (1997).

[19] L. Noonan, B. Doiron, C. Laing, A. Longtin, and R. W. Turner, A Dynamic Dendritic Refractory Period Regulates Burst Discharge in the Electrosensory Lobe of Weakly Electric Fish, J. Neurosci. 23, 1524 (2003).

[20] T. Nevian, M.E. Larkum, A. Polsky, and J. Schiller, Properties of Basal Dendrites of Layer 5 Pyramidal Neurons: A Direct Patch-Clamp Recording Study, Nat. Neurosci. 10, 206 (2007).

[21] P. F. Pinsky and J. Rinzel, Intrinsic and Network Rhythmogenesis in a Reduced Traub Model for $\mathrm{CA}_{3}$ Neurons, J. Comput. Neurosci. 1, 39 (1994).

[22] Z.F. Mainen and T. J. Sejnowski, Influence of Dendritic Structure on Firing Pattern in Model Neocortical Neurons, Nature (London) 382, 363 (1996).

[23] B. Doiron, C. Laing, A. Longtin, and L. Maler, Ghostbursting: A Novel Neuronal Burst Mechanism, J. Comput. Neurosci. 12, 5 (2002).

[24] T. Jarsky, A. Roxin, W. L. Kath, and N. Spruston, Conditional Dendritic Spike Propagation Following Distal Synaptic Activation of Hippocampal CAl Pyramidal Neurons, Nat. Neurosci. 8, 1667 (2005).

[25] P. Poirazi, T. Brannon, and B. W. Mel, Pyramidal Neuron as Two-Layer Neural Network, Neuron 37, 989 (2003).

[26] G. B. Ermentrout, Type I Membranes, Phase Resetting Curves, and Synchrony, Neural Comput. 8, 979 (1996).

[27] P. C. Bressloff and S. Coombes, Desynchronization, Mode Locking, and Bursting in Strongly Coupled Integrate-andFire Oscillators, Phys. Rev. Lett. 81, 2168 (1998).

[28] B. W. Mel, The Clusteron: Toward a Simple Abstraction for a Complex Neuron, in Proceedings of the 4th International Conference on Neural Information Processing Systems (Morgan Kaufmann Publishers Inc., San Francisco, 1992), pp. 35-42.

[29] B. W. Mel, NMDA-Based Pattern Discrimination in a Modeled Cortical Neuron, Neural Comput. 4, 502 (1992). 
[30] P. Poirazi and B. W. Mel, Impact of Active Dendrites and Structural Plasticity on the Memory Capacity of Neural Tissue, Neuron 29, 779 (2001).

[31] M. Jadi, A. Polsky, J. Schiller, and B. W. Mel, LocationDependent Effects of Inhibition on Local Spiking in Pyramidal Neuron Dendrites, PLoS Comput. Biol. 8, e1002550 (2012).

[32] G. R. Yang, J. D. Murray, and X.-J. Wang, A Dendritic Disinhibitory Circuit Mechanism for Pathway-Specific Gating, Nat. Commun. 7, 12815 (2016).

[33] R. Naud, B. Bathellier, and W. Gerstner, Spike-Timing Prediction in Cortical Neurons with Active Dendrites, Front. Comput. Neurosci. 8, 90 (2014).

[34] R.-M. Memmesheimer, Quantitative Prediction of Intermittent High-Frequency Oscillations in Neural Networks with Supralinear Dendritic Interactions, Proc. Natl. Acad. Sci. U.S.A. 107, 11092 (2010).

[35] D. Breuer, M. Timme, and R.-M. Memmesheimer, Statistical Physics of Neural Systems with Nonadditive Dendritic Coupling, Phys. Rev. X 4, 011053 (2014).

[36] Parameters used are $V_{X}=-0.75, \quad V_{Y}=0, T_{R}=0.05$, $b=0.5$, and $D_{Y}=0.016$ unless mentioned otherwise. Unless mentioned otherwise, time is measured in units of the membrane time constant $(\tau=1)$.

[37] G. Ariav, A. Polsky, and J. Schiller, Submillisecond Precision of the Input-Output Transformation Function Mediated by Fast Sodium Dendritic Spikes in Basal Dendrites of $\mathrm{CA}_{1}$ Pyramidal Neurons, J. Neurosci. 23, 7750 (2003).

[38] M. Stemmler, A Single Spike Suffices: The Simplest Form of Stochastic Resonance in Model Neurons, Network 7, 687 (1996).

[39] T. Toyoizumi, K. Aihara, and S. I. Amari, Fisher Information for Spike-Based Population Decoding, Phys. Rev. Lett. 97, 098102 (2006).

[40] C. Shannon, A Mathematical Theory of Communication, Tech. Bell Syst. J. 27, 319 (1948).

[41] W. Bialek, F. Rieke, R. de Ruyter Van Steveninck, and D. Warland, Reading a Neural Code, Science 252, 1854 (1991).

[42] M. J. Chacron, B. Doiron, L. Maler, A. Longtin, and J. Bastian, Non-Classical Receptive Field Mediates Switch in a Sensory Neuron's Frequency Tuning, Nature (London) 423, 77 (2003).

[43] Power spectra involved in the calculation of $\mathcal{M}$ are calculated by averaging 25 segments of $40 \tau$.

[44] The JDP is simulated by adding at every time step of $0.1 \mathrm{~ms}$ a Gaussian random number parametrized by a standard deviation of 0.05 and a mean of 1.04 to a variable randomly jumping between $-a$ and $a$, with $a=0.2$. Jump probability is chosen such that the process will jump on average every $50 \tau$.

[45] N. Parga and L. F. Abbott, Network Model of Spontaneous Activity Exhibiting Synchronous Transitions between Up and Down States, Front. Neurosci. 1, 57 (2007).

[46] A. Destexhe, Self-Sustained Asynchronous Irregular States and Up-Down States in Thalamic, Cortical and Thalamocortical Networks of Nonlinear Integrate-and-Fire Neurons, J. Comput. Neurosci. 27, 493 (2009).

[47] A. Melanson, J. F. Mejias, J. J. Jun, L. Maler, and A. Longtin, Nonstationary Stochastic Dynamics Underlie Spontaneous
Transitions between Active and Inactive Behavioral States, eNeuro 4, ENEURO.0355-16.2017 (2017)..

[48] N. Brunel and J.-P. Nadal, Mutual Information, Fisher Information, and Population Coding, Neural Comput. 10, 1731 (1998).

[49] S. B. Laughlin, R. R.d. R. van Steveninck, and J.C. Anderson, The Metabolic Cost of Neural Information, Nat. Neurosci. 1, 36 (1998).

[50] S. Schreiber, C. K. Machens, A. V. M. Herz, and S. B. Laughlin, Energy-Efficient Coding with Discrete Stochastic Events, Neural Comput. 14, 1323 (2002).

[51] B. Sengupta, M. Stemmler, S. B. Laughlin, and J. E. Niven, Action Potential Energy Efficiency Varies among Neuron Types in Vertebrates and Invertebrates, PLoS Comput. Biol. 6, e1000840 (2010).

[52] B. Ujfalussy, T. Kiss, and P. Erdi, Parallel Computational Subunits in Dentate Granule Cells Generate Multiple Place Fields, PLoS Comput. Biol. 5, e1000500 (2009).

[53] K. Archie and B. Mel, A Model for Intradendritic Computation of Binocular Disparity, Nat. Neurosci. 3, 54 (2000).

[54] M. Murayama, E. Pérez-Garci, T. Nevian, T. Bock, W. Senn, and M. E. Larkum, Dendritic Encoding of Sensory Stimuli Controlled by Deep Cortical Interneurons, Nature (London) 457, 1137 (2009).

[55] L. L. Gollo, O. Kinouchi, and M. Copelli, Active Dendrites Enhance Neuronal Dynamic Range, PLoS Comput. Biol. 5, e1000402 (2009).

[56] N. L. Golding, T. J. Mickus, Y. Katz, W. L. Kath, and N. Spruston, Factors Mediating Powerful Voltage Attenuation Along $\mathrm{CA}_{1}$ Pyramidal Neuron Dendrites, J. Physiol. 568, 69 (2005).

[57] Y. Kubota, F. Karube, M. Nomura, A. T. Gulledge, A. Mochizuki, A. Schertel, and Y. Kawaguchi, Conserved Properties of Dendritic Trees in Four Cortical Interneuron Subtypes, Sci. Rep. 1, 89 (2011).

[58] R. J. Sayer, S. J. Redman, and P. Andersen, Amplitude Fluctuations in Small EPSPs Recorded from $\mathrm{CA}_{1}$ Pyramidal Cells in the Guinea Pig Hippocampal Slice, J. Neurosci. 9, 840 (1989).

[59] J. Waters, M. Larkum, B. Sakmann, and F. Helmchen, Supralinear $\mathrm{Ca}^{2+}$ Influx into Dendritic Tufts of Layer 2/3 Neocortical Pyramidal Neurons In Vitro and In Vivo, J. Neurosci. 23, 8558 (2003).

[60] M. E. Larkum, J. Zhu, and B. Sakmann, A New Cellular Mechanism for Coupling Inputs Arriving at Different Cortical Layers, Nature (London) 398, 338 (1999).

[61] J. C. Magee, Dendritic Ih Normalizes Temporal Summation

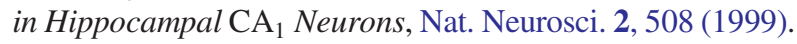

[62] M. E. Larkum, W. Senn, and H.-R. Luscher, Top-Down Dendritic Input Increases the Gain of Layer 5 Pyramidal Neurons, Cereb. Cortex 14, 1059 (2004).

[63] M. Giugliano, G. La Camera, S. Fusi, and W. Senn, The Response of Cortical Neurons to In Vivo-like Input Current: Theory and Experiment: II. Time-Varying and Spatially Distributed Inputs, Biol. Cybern. 99, 303 (2008).

[64] R. Urbanczik and W. Senn, Learning by the Dendritic Prediction of Somatic Spiking, Neuron 81, 521 (2014).

[65] J. Hawkins and S. Ahmad, Why Neurons Have Thousands of Synapses, A Theory of Sequence Memory in Neocortex, Front. Neural Circuits 10 (2016). 
[66] P. Kaifosh and A. Losonczy, Mnemonic Functions for Nonlinear Dendritic Integration in Hippocampal Pyramidal Circuits, Neuron 90, 622 (2016).

[67] S. D. Gale and G. J. Murphy, Active Dendritic Properties and Local Inhibitory Input Enable Selectivity for Object Motion in Mouse Superior Colliculus Neurons, J. Neurosci. 36, 9111 (2016).

[68] R. W. Turner, L. Maler, T. Deerinck, S. R. Levinson, and M. H. Ellisman, TTX-Sensitive Dendritic Sodium Channels Underlie Oscillatory Discharge in a Vertebrate Sensory Neuron, J. Neurosci. 14, 6453 (1994).

[69] N. Takahashi, T. G. Oertner, P. Hegemann, and M. E. Larkum, Active Cortical Dendrites Modulate Perception, Science 354, 1587 (2016).

[70] H. C. Lai and L. Y. Jan, The Distribution and Targeting of Neuronal Voltage-Gated Ion Channels, Nat. Rev. Neurosci. 7, 548 (2006).

[71] S. Mensi, R. Naud, M. Avermann, C. C. H. Petersen, and W. Gerstner, Parameter Extraction and Classification of
Three Neuron Types Reveals Two Different Adaptation Mechanisms, J. Neurophysiol. 107, 1756 (2012).

[72] A. Kamondi, L. Acsády, X.-J. Wang, and G. Buzsáki, Theta Oscillations in Somata and Dendrites of Hippocampal Pyramidal Cells In Vivo: Activity-Dependent PhasePrecession of Action Potentials, Hippocampus 8, 244 (1998).

[73] A. Kepecs, M. van Rossum, S. Song, and J. Tegner, SpikeTiming-Dependent Plasticity: Common Themes and Divergent Vistas, Biol. Cybern. 87, 446 (2002).

[74] J. R. Huguenard, O. P. Hamill, and D. A. Prince, Developmental Changes in $\mathrm{Na}^{+}$Conductances in Rat Neocortical Neurons: Appearance of a Slowly Inactivating Component, J. Neurophysiol. 59, 778 (1988).

[75] O. Hamill, J. Huguenard, and D. Prince, Patch-Clamp Studies of Voltage-Gated Currents in Identified Neurons of the Rat Cerebral Cortex, Cereb. Cortex 1, 48 (1991).

[76] W. Gerstner, W. Kistler, R. Naud, and L. Paninski, Neuronal Dynamics (Cambridge University Press, Cambridge, England, 2014). 